\title{
Severe Pancytopenia Induced by Valproic Acid
}

\author{
Andrew Wahba ${ }^{1}$, Emmalee Bergez ${ }^{1}$ \\ 1. Pediatrics, McGovern Medical School, University of Texas Health Science Center at Houston, Houston, USA
}

Corresponding author: Andrew Wahba, andrew.a.wahba@uth.tmc.edu

\begin{abstract}
Valproic acid is commonly used to treat pediatric epilepsy. This drug is usually well-tolerated; its side effects are typically mild, with hepatotoxicity being the most widely recognized one. Bone marrow suppression is a rarely seen complication in patients with valproic acid levels more than $125 \mathrm{mcg} / \mathrm{mL}$. Reported cases indicate an increased incidence of hematologic toxicity; however, evidence for management is limited. We report a case of bone marrow suppression induced by a high dose of valproic acid in a 10-year-old male.
\end{abstract}

Categories: Neurology, Pediatrics, Hematology

Keywords: bone marrow suppression, pancytopenia, valproic acid

\section{Introduction}

Valproic acid (VPA) is the most commonly used anticonvulsant, initially approved by the U.S. Food and Drug Administration (FDA) in 1978 to be used as a monotherapy or adjunctive therapy for complex partial and absence seizures. Recently, it has been approved for use in bipolar disorder and migraine prophylaxis. VPA is a small-branched chain fatty acid that is highly protein-bound (>85\%) in a saturable manner, which results in lower clearance rate and urine excretion. The percentage of protein binding decreases with higher VPA levels. VPA decreases neuronal hyperexcitability through several mechanisms. These include increasing the inhibitory neurotransmitter gamma-aminobutyric acid (GABA) by inhibiting its degradation and increasing synthesis, blockage of the calcium channel voltage-dependent, inhibiting the synthesis of inositol, and modulating glutamatergic transmission [1,2]. VPA is mainly metabolized in the liver through microsomal glucuronidation, which becomes fully effective by the age of four years, mitochondrial beta-oxidation, and, to a lesser extent, cytochrome P450-dependent oxidation [3]. It has an average elimination half-life of 4-16 hours depending on patient age and combined use with enzyme-inducing agents such as phenytoin, carbamazepine, and barbiturates $[4,5]$. Plasma clearance is $50 \%$ higher in the age of 2-10 years compared to adults [6]. VPA has a wide therapeutic trough level concentration between 50 and $100 \mathrm{mcg} / \mathrm{mL}$ for seizures and $125 \mathrm{mcg} / \mathrm{mL}$ for manic episodes in bipolar disease. Therapeutic daily dose ranges between 15 and 60 $\mathrm{mg} / \mathrm{kg}$. VPA is usually well-tolerated; its side effects are typically mild, with hepatotoxicity being the most widely recognized one. Hepatotoxicity usually happens during the first six months of treatment; children under the age of 2 years are at higher risk of developing fatal hepatotoxicity. Other known side effects are pancreatitis, hyperammonemia, hypothermia, suicidal ideations, and birth defects particularly neural tube defects. However, as levels exceed $125 \mathrm{mcg} / \mathrm{mL}$, few cases of hematologic toxicity such as thrombocytopenia, platelet dysfunction, neutropenia, pure red cell aplasia, and acute leukemia have been reported. In this report, we identify a case of severe pancytopenia induced by VPA in a pediatric patient.

\section{Case Presentation}

An 11-year-old Hispanic male with a history of autism spectrum disorder (ASD), Dravet syndrome due to SCN1A gene mutation, and intractable epilepsy presented with five days of lethargy, decreased oral intake, and seven pounds weight loss. The patient's mother denied any fever, abdominal pain, vomiting, headache, or sick contacts. His last seizure was six months prior. He previously failed multiple anti-seizure medications such as levetiracetam and clobazam. He had a vagus nerve stimulator placed at the age of five years. He was developmentally delayed with speech and learning difficulties. He had no past medical history of any hematologic disorders. Family history was negative for seizures, developmental, or bleeding disorders. The patient was taking VPA $31 \mathrm{mg} / \mathrm{kg} / \mathrm{day}$ and lacosamide $7 \mathrm{mg} / \mathrm{kg} /$ day for seizure control. He had been on this regimen for the past eight years without any recent new medications or changes.

On physical examination, oral temperature was $98.1^{\circ} \mathrm{F}$, heart rate was 85 beats per minute, blood pressure was $97 / 60 \mathrm{mmHg}$, respiratory rate was 20 breaths per minute, and oxygen saturation was $99 \%$ on room air. Weight was $28.9 \mathrm{~kg}$, with a body mass index (BMI) of $16.59 \mathrm{~kg} / \mathrm{m}^{2}$. The abdomen was soft, non-distended, non-tender with no hepatosplenomegaly. Heart and lung exams were clear, and no skin rash or lesions were seen. On a neurological exam, he was awake and followed commands, and was able to move all extremities with intact cranial nerves, sensations, and reflexes. No jaundice, oral ulcers, thrush, lymphadenopathy, petechiae, ecchymosis, or signs of bleeding were present.

In the emergency room, lab workup was notable for pancytopenia (Table 1), with a VPA level of $255 \mathrm{mcg} / \mathrm{mL}$ (therapeutic range: $50-125 \mathrm{mcg} / \mathrm{mL}$ ). This VPA level was drawn approximately three hours after the last dose 
was given. VPA was discontinued, topiramate was initiated with lacosamide for proper seizure prophylaxis, and the patient was admitted for further workup of pancytopenia. Reticulocyte count on admission was 1.5 $\%$, demonstrating inadequate bone marrow response to pancytopenia. On the following day, the patient developed conjunctival pallor and bilateral lower extremities petechiae. Vital signs remained within normal limits. On repeat lab tests, the patient had worsening pancytopenia (Table 1), with an absolute neutrophil count (ANC) of 300/ $\mathrm{mm}^{3}$. Due to concerns for altered mental status in the setting of severe thrombocytopenia, a CT of the head was obtained, which showed no signs of intracranial bleeding. The peripheral smear showed evidence of thrombocytopenia and leukopenia, but no blasts or other concerns for leukemia were identified.

\begin{tabular}{|c|c|c|c|c|c|c|c|}
\hline Lab Test & Day 1 & Day 2 & Day 4 & Day 5 & Day 6 & Day 8 & Day 9 \\
\hline Valproic acid (mcg/mL) & 255 & 214 & 102 & 53 & 31 & 6 & \\
\hline WBC $\times 10^{3} / \mathrm{mm}^{3}$ & 5.1 & 3.6 & 3.6 & 2.3 & 4.9 & 6.8 & 7 \\
\hline $\mathrm{RBC} \times 10^{6} / \mathrm{mm}^{3}$ & 3 & 2.2 & 2.2 & 1.7 & 3.2 & 3.2 & 3.1 \\
\hline Hemoglobin (g/dL) & 10.7 & 7.9 & 7.9 & 6.4 & 11.2 & 11.3 & 11 \\
\hline Hematocrit (\%) & 31.9 & 22.6 & 23.1 & 18.4 & 32.5 & 33.2 & 31.1 \\
\hline Platelet $\times 10^{3} / \mathrm{mm}^{3}$ & 10 & 4 & 7 & 3 & 124 & 103 & 143 \\
\hline MPV (fL) & 9.7 & 9.4 & 9.2 & 8.2 & 7.8 & 9.4 & 9.5 \\
\hline Neutrophil $\times 10^{3} / \mathrm{mm}^{3}$ & 0.9 & 0.3 & 0.7 & 0.4 & 1.7 & 1.6 & 1.5 \\
\hline Monocytes $\times 10^{3} / \mathrm{mm}^{3}$ & 0.3 & 0.1 & 0.4 & 0.8 & 1.4 & 1.6 & 1.2 \\
\hline Lymphocyte $\times 10^{3} / \mathrm{mm}^{3}$ & 3.9 & 3.2 & 2.5 & 1.6 & 2.3 & 3.6 & 4.2 \\
\hline Reticulocyte count (\%) & 1.5 & & & & 3.6 & 5.4 & 4.7 \\
\hline ALT (unit/L) & & 12 & & & 17 & 25 & \\
\hline AST (unit/L) & & 22 & & & 45 & 64 & \\
\hline Ammonia ( $\mu \mathrm{g} / \mathrm{dL})$ & & 147 & 103 & 79 & 66 & 78 & 74 \\
\hline Total bilirubin (mg/dL) & & 0.8 & & & 0.7 & 0.5 & \\
\hline Albumin (g/dL) & & 3 & & & 2.9 & 2.9 & \\
\hline Sodium (mEq/L) & 140 & 141 & & & 140 & 139 & \\
\hline Potassium (mEq/L) & 3.7 & 3.9 & & & 4.2 & 4 & \\
\hline Creatinine (mg/dL) & 0.50 & 0.43 & & & 0.42 & 0.39 & \\
\hline BUN (mg/dL) & 15 & 13 & & & & 16 & \\
\hline
\end{tabular}

\section{TABLE 1: Laboratory values at different time points}

WBC, white blood cell; RBC, red blood cell; MPV, mean platelet volume; ALT, alanine aminotransferase; AST, aspartate aminotransferase; BUN, blood urea nitrogen

The underlying reason for elevated VPA levels was unclear despite the patient's medication compliance. Since VPA is mainly metabolized in the liver, factor $\mathrm{V}$ level was obtained to assess liver function, which returned within normal limits, and abdominal ultrasound showed normal size liver with normal echogenicity. The patient had previous outpatient liver enzyme levels that were within normal limits, the most recent of which was six months prior.

A trial of intravenous immunoglobulin (IVIG) $1 \mathrm{~g} / \mathrm{kg}$ was given on admission day 3 to determine if the process was immune-mediated. However, follow-up laboratory values continued to show pancytopenia despite the VPA level progressively decreasing to $102 \mathrm{mcg} / \mathrm{mL}$. To rule out other causes of bone marrow aplasia, Epstein-Barr virus (EBV), cytomegalovirus (CMV), human immunodeficiency virus (HIV), and parvovirus B19 serology were negative. He had normal vitamin B12, erythrocyte sedimentation rate (ESR), lactate dehydrogenase (LDH), haptoglobin, and D-dimer levels. Neutrophil antibody was undetected by flow 
cytometry.

Due to persistent anemia and severe thrombocytopenia, a single transfusion of $10 \mathrm{~mL} / \mathrm{kg}$ packed red blood cells and $10 \mathrm{~mL} / \mathrm{kg}$ platelets were given for supportive measures on admission day 5 . On the following days, the patient's laboratory values showed evidence of gradual bone marrow recovery, and the patient was discharged on day 9.

\section{Discussion}

We report a case of a pediatric patient with a history of intractable epilepsy and SCN1A mutation causing Dravet syndrome who has been receiving VPA for eight years with no reported side effects. He presented with toxic VPA levels and severe pancytopenia. VPA affected all the three bone marrow cell lines, which started to recover around day 6 after discontinuation of the drug.

SCN1A gene, located on chromosome 2q24, is one of the most commonly known epilepsy genes. Our patient has a missense variant c.1096G>C; p.Asp366His (one guanine ribonucleotide was altered to cytosine in codon 1096, which caused a change in the reading frame from aspartate to histidine). SCN1A mutations have been linked to multiple epilepsy disorders such as Dravet syndrome, which is also known as severe myoclonic epilepsy in infancy (MIM\# 607208). Dravet syndrome usually presents as a refractory seizure during the first year of life and developmental delay. SCN1A mutations impair the inhibition of neuronal sodium channel activity, which increases neuronal excitability $[7,8]$.

Our patient had severe pancytopenia, hyperammonemia with normal liver enzymes, and kidney function levels. L-carnitine has been shown to be effective in VPA-induced hepatotoxicity and hyperammonemia above $80 \mu \mathrm{g} / \mathrm{dL}$ [9]. Exogenous carnitine binds to VPA, enhancing the beta-oxidation and urea synthesis process, thus decreasing ammonia levels. Since the repeat ammonia level for our patient dropped below 80 $\mu \mathrm{g} / \mathrm{dL}$, the decision was to trend the ammonia level without giving carnitine.

Upon admission, differential diagnoses for pancytopenia included drug-induced myelosuppression, autoimmune-mediated pancytopenia, and other causes of bone marrow failure such as idiopathic acquired aplastic anemia, hypoplastic myelodysplastic syndrome, infections, nutritional deficiencies, hematopoietic and lymphoid neoplasms, and myelofibrosis. Peripheral smear findings, negative viral serology, negative neutrophil antibody, poor response to IVIG, and spontaneous recovery after discontinuation of VPA support that the cause was most likely drug-induced bone marrow suppression.

VPA can cause a wide spectrum of hematologic toxicities such as thrombocytopenia, acquired Von Willebrand disease, neutropenia, Pelger-Huet anomalies, macrocytosis, pure red cell aplasia, and acute leukemia. These toxicities appear to be reversible and dose-related with the female gender being at higher risk $[10,11]$. Mild thrombocytopenia is the most commonly reported toxicity, and it usually does not require immediate discontinuation of the drug [12]. Few cases of severe pancytopenia have been reported. Direct bone marrow suppression and immune-mediated destruction are the two known mechanisms. In an in vitro study to assess direct bone marrow suppression, VPA markedly inhibited the growth of bone marrow progenitor cells at a concentration of $200 \mathrm{mcg} / \mathrm{mL}$, and normal growth was seen at a concentration of 100 $\mathrm{mcg} / \mathrm{mL}$ [13]. We believe the patient's supra-therapeutic VPA levels have led to the severity of his symptoms. Management is mainly supportive through discontinuing VPA in severe cases or lowering the daily dose in mild cases, use of IVIG in immune-mediated toxicity, desmopressin or aminocaproic acid in patients with bleeding diathesis, granulocyte colony-stimulating factor (GCSF) in neutropenic patients, and blood transfusion in life-threatening conditions $[14,15]$.

\section{Conclusions}

VPA-induced hematologic toxicity is becoming more frequently encountered; however, evidence for management is limited. Bone marrow suppression is a rarely seen complication in patients with VPA levels > $125 \mathrm{mcg} / \mathrm{mL}$. In these patients, immediate discontinuation of the drug is recommended. Resolution of the bone marrow suppression is expected to occur within 10 days of VPA withdrawal. Patients taking VPA may benefit from periodic monitoring of cell line counts, and caution should be taken when prescribing high doses.

\section{Additional Information}

\section{Disclosures}

Human subjects: Consent was obtained by all participants in this study. Conflicts of interest: In compliance with the ICMJE uniform disclosure form, all authors declare the following: Payment/services info: All authors have declared that no financial support was received from any organization for the submitted work. Financial relationships: All authors have declared that they have no financial relationships at present or within the previous three years with any organizations that might have an interest in the submitted work. Other relationships: All authors have declared that there are no other relationships or activities that could appear to have influenced the submitted work. 


\section{References}

1. Perucca E: Pharmacological and therapeutic properties of valproate: a summary after 35 years of clinical experience. CNS Drugs. 2002, 16:695-714. 10.2165/00023210-200216100-00004

2. Löscher W: Basic pharmacology of valproate: a review after 35 years of clinical use for the treatment of epilepsy. CNS Drugs. 2002, 16:669-94. 10.2165/00023210-200216100-00003

3. Ghodke-Puranik Y, Thorn CF, Lamba JK, et al.: Valproic acid pathway: pharmacokinetics and pharmacodynamics. Pharmacogenet Genomics. 2013, 4:236-41. 10.1097/FPC.0b013e32835ea0b2

4. Dutta S, Reed RC: Functional half-life is a meaningful descriptor of steady-state pharmacokinetics of an extended-release formulation of a rapidly cleared drug: as shown by once-daily divalproex-ER. Clin Drug Investig. 2006, 26:681-90. 10.2165/00044011-200626120-00002

5. Williams JH, Jayaraman B, Swoboda KJ, Barrett JS: Population pharmacokinetics of valproic acid in pediatric patients with epilepsy: considerations for dosing spinal muscular atrophy patients. J Clin Pharmacol. 2012, 52:1676-88. 10.1177/0091270011428138

6. Guerrini R: Valproate as a mainstay of therapy for pediatric epilepsy . Paediatr Drugs. 2006, 8:113-29. 10.2165/00148581-200608020-00004

7. Martin MS, Dutt K, Papale LA, et al.: Altered function of the SCN1A voltage-gated sodium channel leads to gamma-aminobutyric acid-ergic (GABAergic) interneuron abnormalities. J Biol Chem. 2010, 26:9823-34. 10.1074/jbc.M109.078568

8. Escayg A, Goldin AL: Sodium channel SCN1A and epilepsy: mutations and mechanisms. Epilepsia. 2010, 51:1650-8. 10.1111/i.1528-1167.2010.02640.x

9. Lheureux PE, Penaloza A, Zahir S, Gris M: Science review: carnitine in the treatment of valproic acidinduced toxicity - what is the evidence?. Crit Care. 2005, 5:431-40. 10.1186/cc3742

10. Nasreddine W, Beydoun A: Valproate-induced thrombocytopenia: a prospective monotherapy study . Epilepsia. 2008, 49:438-45. 10.1111/j.1528-1167.2007.01429.x

11. Buoli M, Serati M, Botturi A, Altamura AC: The risk of thrombocytopenia during valproic acid therapy: a critical summary of available clinical data. Drugs RD. 2018, 18:1-5. 10.1007/s40268-017-0224-6

12. Allarakhia IN, Garofalo EA, Komarynski MA, Robertson PL: Valproic acid and thrombocytopenia in children: a case-controlled retrospective study. Pediatr Neurol. 1996, 14:303-7. 10.1016/0887-8994(96)00052-5

13. Kishi T, Fujita N, Kawaguchi H, Ishimae M, Watanabe K, Tanaka T: Bone marrow suppression induced by high dose valproic acid. Arch Dis Child. 1994, 71:153-5. 10.1136/adc.71.2.153

14. Acharya S, Bussel JB: Hematologic toxicity of sodium valproate. J Pediatr Hematol Oncol. 2000, 22:62-5. 10.1097/00043426-200001000-00012

15. Vesta KS, Medina PJ: Valproic acid-induced neutropenia. Ann Pharmacother. 2003, 37:819-21. 10.1345/aph.1C381 